\title{
Genome-Wide Identification and
} Characterization of HSP90-RAR1-SGT1-Complex Members From Arachis Genomes and Their Responses to Biotic and Abiotic Stresses

\author{
Cuiling Yuan, Chunjuan Li, Xiaobo Zhao, Caixia Yan, Juan Wang, Yifei Mou, Quanxi Sun* \\ and Shihua Shan*
}

Shandong Peanut Research Institute, Qingdao, China

\section{OPEN ACCESS}

Edited by:

Nidhi Rawat,

University of Maryland, College Park,

United States

Reviewed by:

Frank Maulana,

Louisiana State University Agricultural

Center, United States

Gyan P. Mishra,

Indian Agricultural Research Institute

(ICAR), India

*Correspondence:

Quanxi Sun

squanxi@163.com

Shihua Shan

shansh1971@163.com

Specialty section: This article was submitted to

Plant Genomics,

a section of the journal

Frontiers in Genetics

Received: 01 April 2021 Accepted: 05 August 2021

Published: 27 August 2021

Citation:

Yuan C, Li C, Zhao X, Yan C Wang J, Mou Y, Sun Q and Shan S (2021) Genome-Wide Identification and Characterization of HSP90-RAR1-SGT1-Complex Members From Arachis Genomes and Their Responses to Biotic and Abiotic Stresses.

Front. Genet. 12:689669. doi: 10.3389/fgene.2021.689669
The molecular chaperone complex HSP90-RAR1-SGT1 (HRS) plays important roles in both biotic and abiotic stress responses in plants. A previous study showed that wild peanut Arachis diogoi SGT1 (AdSGT1) could enhance disease resistance in transgenic tobacco and peanut. However, no systematic analysis of the HRS complex in Arachis has been conducted to date. In this study, a comprehensive analysis of the HRS complex were performed in Arachis. Nineteen HSP90, two RAR1 and six SGT1 genes were identified from the allotetraploid peanut Arachis hypogaea, a number close to the sum of those from the two wild diploid peanut species Arachis duranensis and Arachis ipaensis. According to phylogenetic and chromosomal location analyses, thirteen orthologous gene pairs from Arachis were identified, all of which except AhHSP9OA8, AhHSP90-B9, AdHSP90-9, and AiHSP90-9 were localized on the syntenic locus, and they shared similar exon-intron structures, conserved motifs and expression patterns. Phylogenetic analysis showed that HSP90 and RAR1 from dicot and monocot plants diverged into different clusters throughout their evolution. Chromosomal location analysis indicated that AdSGT1 (the orthologous gene of AhSGT1-B3 in this study) might provide resistance to leaf late spot disease dependent on the orthologous genes of AhHSP90-B10 and AhRAR1-B in the wild peanut $A$. diogoi. Several HRS genes exhibited tissue-specific expression patterns, which may reflect the sites where they perform functions. By exploring published RNA-seq data, we found that several HSP9O genes play major roles in both biotic and abiotic stress responses, especially salt and drought responses. Autoactivation assays showed that AhSGT1-B1 could not be used as bait for yeast two-hybrid $(\mathrm{Y} 2 \mathrm{H})$ library screening. AhRAR1 and AhSGT1 could strongly interact with each other and interact with AhHSP90-B8. The present study represents the first systematic analysis of HRS complex genes in Arachis and provides valuable information for functional analyses of HRS complex genes. This study also offers potential stress-resistant genes for peanut improvement.

Keywords: HSP90-RAR1-SGT1 complex, molecular characteristics, stress-responsive, protein interaction, Arachis 


\section{INTRODUCTION}

Peanut, also known as groundnut (Arachis hypogaea L.), is an economically important, nutritious and protein-rich oilseed crop species grown around the world (Zhuang et al., 2019). Abiotic stresses such as salt (Zhang et al., 2020), drought (Zhao et al., 2018), calcium deficiency (Chen et al., 2019) and biotic stresses such as Aspergillus flavus contamination (Zhao et al., 2020) strongly affect peanut quality and yield. Breeding stress-resistant peanut cultivars is an effective way to avoid declines in quality and yield. The mining and characterization of stress-responsive genes or gene families can facilitate crop quality improvement. However, few stress-responsive genes in peanut have been identified. In recent years, genome information (Bertioli et al., 2016; Zhuang et al., 2019) and many RNA-seq data (Clevenger et al., 2016; Zhao et al., 2018, 2020; Chen et al., 2019; Zhang et al., 2020) for peanut have become available, which are convenient for gene family characterization and mining stressresponsive genes.

Plants have evolved stress-resistance genes to cope with abiotic and biotic stresses. These genes include the three members of the HSP90-RAR1-SGT1 (HRS) complex, namely, HSP90 (heat shock protein 90), its chaperone RAR1 (required for MLA12 resistance) and SGT1 [suppressor of the G2 allele of SKP1 (S-phase kinaseassociated protein 1)] (Shirasu, 2009). The HRS complex plays important roles in plant resistance to disease by stabilizing many NLR proteins. RAR1 was originally cloned from barley and encodes a protein containing two highly conserved zincbinding domains, CHORD1 and CHORD2 (Shirasu et al., 1999). SGT1 was initially identified as involved in the yeast kinetochore assembly pathway (Krishna and Gloor, 2001) and contains five regions: a tetratricopeptide repeat (TPR) domain, two variable regions (VR1 and VR2), the CS (CHORD-containing protein and SGT1) domain, and the SGS (SGT1-specific motif) domain. The HSP90 family is a highly conserved molecular chaperone that plays diverse roles in plants (Krishna and Gloor, 2001; Kadota and Shirasu, 2011). It consists of three segments: an N-terminal ATPase domain $(\mathrm{N})$, a substrate-binding domain in the middle (M), and a C-terminal dimerization domain (Kadota and Shirasu, 2011). HSP90, RAR1, and SGT1 can interact with each other, and their interaction is essential for their functions in biotic stress resistance (Shirasu, 2009; Ito et al., 2015). HRS-related genes are also involved in the abiotic stress response (Reddy et al., 2011; Shanmugam et al., 2016; Zhang et al., 2017; Vishwakarma et al., 2018). E. coli expressing Pennisetum glaucum HSP90 exhibits enhanced tolerance to heat, salt and dehydration stresses (Reddy et al., 2011). Overexpression of the rice Hsp90 gene can significantly increase the salt stress response of transgenic tobacco (Liu et al., 2006). The BolSGT1 gene can potentially improve B. oleracea resistance to abiotic stresses such as heat, cold, and salt (Shanmugam et al., 2016). The expression of barley HSP90 genes has been shown to be upregulated under heat stress, heavy metal stress and calcium chloride treatment (Chaudhary et al., 2019). However, it seems that RAR1 is not involved in the abiotic stress response.

HRS complex-related genes have been identified in many plant species (Krishna and Gloor, 2001; Zhang et al., 2013, 2017;
Agarwal et al., 2016; Song et al., 2019), for example, 7 in Arabidopsis (Krishna and Gloor, 2001) and 9 in rice (Hu et al., 2009). In common wheat, TaHSP90 genes encode three types of HSP90 proteins (TaHSP90.1, TaHSP90.2, and TaHSP90.3) (Wang et al., 2011), and each TaHSP90 has three homologous genes in each genome (for example, TaHSP90.1-A1, TaHSP90.1B1, and TaHSP90.1-C1). Five HSP90 genes in chickpea, seven in pigeonpea, six in common bean, and five in Medicago and Lotus have been identified (Agarwal et al., 2016). RAR1 exists widely in plants but is absent in Chlamydomonas (Shirasu, 2009). Three distinct RAR1 homeologs (TaRAR1-A1, TaRAR1-B1, and TaRAR1-D1) have been isolated from common wheat (Wang et al., 2015). Arabidopsis contains two SGT1 isoforms, SGT1a and SGT1b (Austin et al., 2002). Three different SGT1 homeologs (TaSGT1-A1, TaSGT1-B1, and TaSGT1-D1) have been identified in common wheat (Wang et al., 2015).

The pathogen-induced SGT1 of wild peanut Arachis diogoi can induce cell death and enhance disease resistance in transgenic tobacco and peanut (Kumar and Kirti, 2015). However, the understanding of HRS-related genes in Arachis is still very poor. In this study, HRS-related genes were identified from both wild peanuts and cultivated peanuts. We also conducted chromosomal location, phylogenetic, gene structure, conserved motif, and tissue expression profiling analyses. In addition, expression profiling of HRS-related genes under abiotic and biotic stresses in cultivated peanut was performed. Furthermore, probable protein interactions among the RAR1, SGT1, and HSP90 genes were investigated.

\section{MATERIALS AND METHODS}

\section{Identification of HRS-Related Proteins in Arachis Genomes}

All HRS-related proteins were obtained from the PeanutBase database $^{1}$ (Dash et al., 2016). These putative candidate proteins were manually verified using $\mathrm{NCBI}^{2}$ to confirm the presence of conserved motifs (CHORD1, CCCH, and CHORD2 for RAR1 proteins; TPR, CS, and SGS for SGT1 proteins; HATPase_c, HSP90 and MEEVD for HSP90 proteins). To verify the reliability of the search results, each protein sequence was examined using the domain analysis program SMART (Simple Modular Architecture Research $\mathrm{Tool}^{3}$ ) and the PFAM (Protein family) database ${ }^{4}$. Only the sequences containing these domains were retained. The protein isoelectric points (pIs) and molecular weights (MWs) were predicted using proteomics and sequence analysis tools on the ExPASy Proteomics Server ${ }^{5}$. The putative Arabidopsis orthologs for peanut HRS-related proteins were identified using a BLASTp search.

\footnotetext{
${ }^{1} \mathrm{http}: / /$ peanutbase.org/

${ }^{2}$ https://www.ncbi.nlm.nih.gov/

${ }^{3} \mathrm{http}: / /$ smart.embl-heidelberg.de/

${ }^{4}$ http://pfam.xfam.org/

${ }^{5}$ http://web.expasy.org/compute_pi/
} 


\section{Chromosomal Location, Gene Structure and Conserved Motif Analyses}

The chromosomal location information of HRS-related proteins was obtained from the PeanutBase website (see text footnote 1) (Dash et al., 2016). The genes were mapped onto the chromosomes using the MapInspect software program ${ }^{6}$. The Gene Structure Display Server (GSDS) program ( ${ }^{7} \mathrm{Hu}$ et al., 2015) was used to elucidate the exon/intron organization of HRSrelated genes. The Multiple Em for Motif Elicitation (MEME) program ( ${ }^{8}$ Bailey et al., 2009) was used to illustrate the motifs in HRS-related protein sequences.

\section{Sequence Alignment and Phylogenetic Analyses}

The representative HSP90, RAR1, and SGT1 gene sequences from different species were retrieved from the NCBI database. Multiple alignment of their predicted amino acid sequences were performed using the computer program ClustalW (Larkin et al., 2007). Unrooted phylogenetic trees were constructed according to the neighbor-joining (NJ) method using MEGA 6.0 software (Tamura et al., 2013), and the bootstrap test was carried out with 1000 iterations.

\section{RNA-Seq-Based Expression Profiling of NAC Genes in Peanut}

Gene expression data from 22 different tissues were obtained from Clevenger et al. (2016). To explore genes responsive to biotic and abiotic stresses, transcriptome sequencing information under salt, drought, low calcium, and Aspergillus flavus infection treatments was retrieved from the Short Read Archive at NCBI, with accession numbers SRR8177741 (Zhang et al., 2020), SRP093341 (Zhao et al., 2018), SRS3289849 (Chen et al., 2019), and PRJNA438019 (Zhao et al., 2020), respectively. The FPKM data or fold-change values for each HRS gene were $\log 2$ transformed and displayed in the form of heatmaps via HemI (Deng et al., 2014).

\section{Peanut Materials and Growth Conditions}

Huayu 9303, a peanut cultivar with high yield and good quality, was grown in a temperature-controlled chamber at $20^{\circ} \mathrm{C}$ with a photoperiod of $16 \mathrm{~h}$ light and $8 \mathrm{~h}$ dark unless otherwise stated. After approximately 1 month, the leaves were collected, frozen immediately in liquid nitrogen and stored at $-80^{\circ} \mathrm{C}$.

\section{Gene Cloning and Plasmid Construction}

Several HRS-related genes from the cultivated peanut Huayu 9303 were isolated and then subcloned them into plasmids for yeast two-hybrid $(\mathrm{Y} 2 \mathrm{H})$ assays. Total RNA was isolated using TRIzol reagent (Invitrogen). To clone the full-length coding regions of these genes, reverse transcription polymerase chain reaction (RT-PCR) was performed according to the M-MLV Reverse Transcriptase (Invitrogen) protocol. The amplification

\footnotetext{
${ }^{6} \mathrm{http}: / /$ mapinspect.software.informer.com

${ }^{7}$ http://gsds.cbi.pku.edu.cn

${ }^{8} \mathrm{http}: / /$ meme-suit.org
}

of all genes was performed with an initial denaturation at $98^{\circ} \mathrm{C}$ for $30 \mathrm{~s}$, followed by 30 cycles at $98^{\circ} \mathrm{C}$ for $10 \mathrm{~s}, 58^{\circ} \mathrm{C}$ for $10 \mathrm{~s}$, and $72^{\circ} \mathrm{C}$ for $1 \mathrm{~min}$, and a final extension at $72^{\circ} \mathrm{C}$ for $10 \mathrm{~min}$. PCR amplifications were performed using Phusion High Fidelity DNA polymerase (New England Biolabs). PCR products were first cloned into the pENTR/D TOPO vector (Invitrogen) and sequenced. The cloned genes were then recombined into the Gateway-compatible pGADT7 (AD) and pGBKT7 (BD) vectors for $\mathrm{Y} 2 \mathrm{H}$ analysis.

\section{Y2H Analysis}

For $\mathrm{Y} 2 \mathrm{H}$ autoactivation and pairwise interaction assays, we implemented the Gal4-based Matchmaker Gold Yeast Two-Hybrid System (Clontech ${ }^{9}$ ). This system includes four independent reporters that allow testing interactions at different stringencies, with the use of a double dropout medium (DDO: SD/-Trp/-Leu), triple dropout medium (TDO: SD/Trp/-Leu/-His), and quadruple dropout medium (QDO: $\mathrm{SD} /$-Trp/-Leu/-His/-Ade) (Clontech). The plasmids BD-wXb12 and $\mathrm{AD}-w \mathrm{Xb} 12 \mathrm{IP} 2$ were used as positive controls, and the interaction between $\mathrm{BD}-\mathrm{wXb12}$ and $\mathrm{AD}-\mathrm{wRAR} 1$ served as a negative control (Pei et al., 2015).

\section{RESULTS}

\section{Identification and Characterization of HSP90 Genes}

Ten HSP90 genes were identified from the genomes of the diploid wild peanut species A. duranensis and A. ipaensis. Because of the lack of a nomenclatural method for HSP90 genes, we named these genes AdHSP90-1 to AdHSP90-10 and AiHSP90-1 to AiHSP90-10 according to their arrangement on the chromosome (Figure 1). The HSP90 genes identified in this study had MWs ranging from 73.60 to $92.41 \mathrm{kDa}$. The encoded proteins varied from 646 amino acids (aa) to 815 aa in length, with an average of 732.9 aa. The pIs of the predicted proteins were determined as shown in Table 1. In the allotetraploid cultivated peanut A. hypogaea, 9 and $10 \mathrm{HSP} 90$ genes were localized on the A and B subgenomes, respectively. We named them AhHSP90-A1 to AhHSP90-A9 and AhHSP90-B1 to AhHSP90-B10. One HSP90 gene was lost in the A subgenome of cultivated peanut. The size of the HSP90 proteins in A. hypogaea ranged from 340 to 812 aa, with an average of 711.8 aa (Table 1).

Chromosomal location analysis showed that all 39 HSP90 genes were randomly distributed on every chromosome except chromosomes A4, A6, A10, B6, B8, and B10 of A. duranensis and $A$. ipaensis (Figure 1) and chromosomes 4, 6, 10, 14, 16, 18, and 20 of cultivated peanut (Figure 2). Orthologous gene pairs (Table 2) were obtained according to the phylogenetic analysis results (Figure 3). In general, orthologous genes were located on the syntenic locus of each chromosome. AdHSP90-9 and AhHSP90-A8 localized on chromosome A8 of A. duranensis and chromosome 7 of $A$. hypogaea, while their orthologous genes AiHSP90-9 and AhHSP90-B9 were assigned to chromosomes

${ }^{9} \mathrm{http}: / /$ www.clontech.com/ 


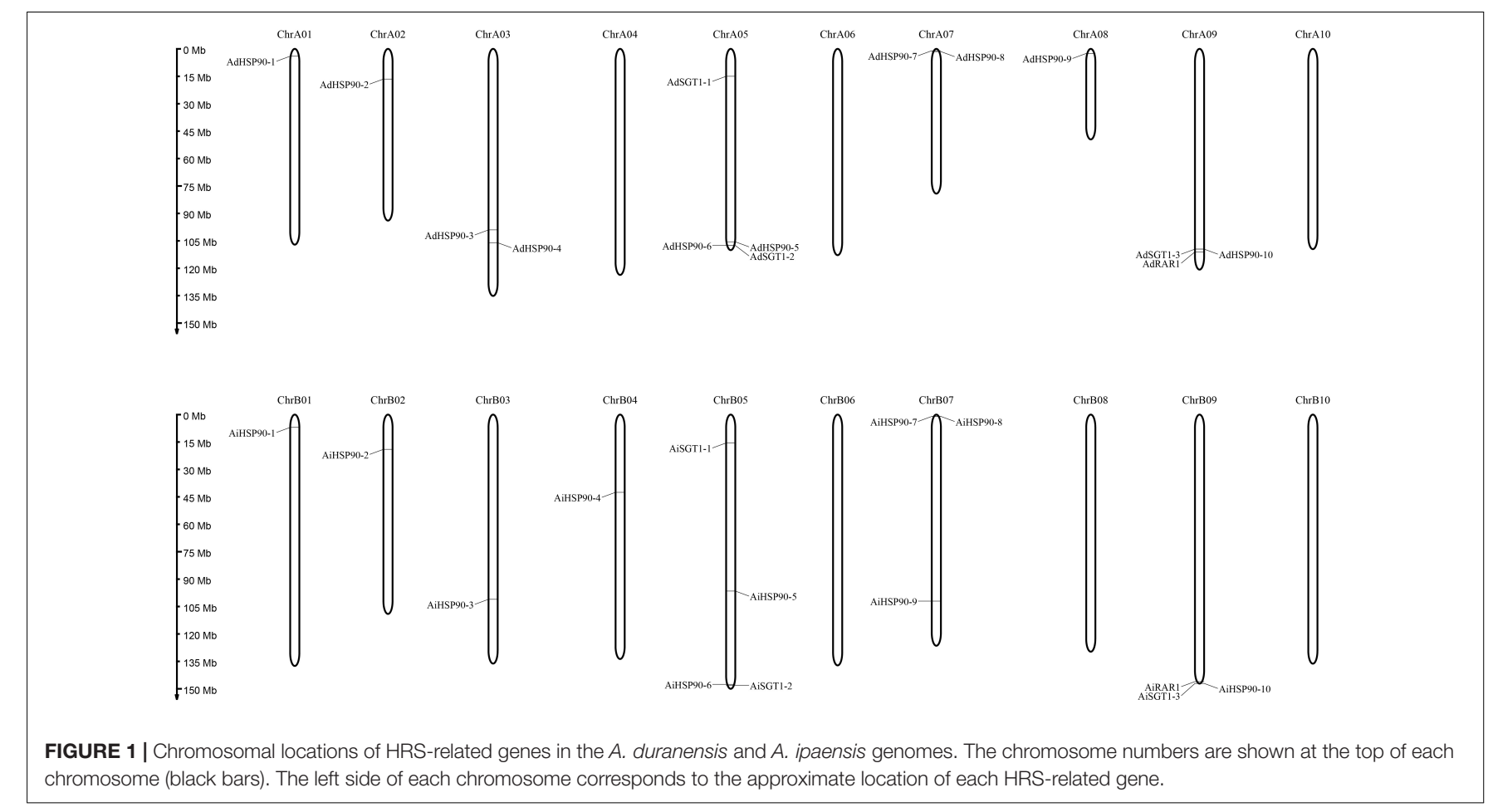

B8 and 17, respectively. The orthologous gene pairs from the same genome of wild and cultivated peanut (for example, A. duranensis and the A subgenome of A. hypogaea) usually shared high sequence similarity (Table 2). Some orthologous genes shared identical protein sequences; for example, AdHSP901 and AhHSP90-A1 shared the same amino acid sequence; however, their CDSs were slightly different. Most orthologous genes shared similar exon-intron structures and conserved motifs, for example, AdHSP90-3, AiHSP90-3, AhHSP90-A3, and AhHSP90-B3 (Figure 4). Most HSP90 genes contained 3 or 4 exons, while some had more than 19 exons, which might explain the diversity of HSP90 functions. Remarkably, AdHSP904 contained 20 exons; however, there was only one motif in its putative protein sequence.

\section{Identification and Characterization of RAR1 Genes}

Only one RAR1 gene was identified from each diploid, A. duranensis and A. ipaensis, and was named AdRAR1 and $A i R A R 1$, respectively. The deduced protein sequences of $A d R A R 1$ and AiRAR1 contained 277 and 271 amino acid residues, respectively (Table 1). The AdRAR1 and AiRAR1 genes were assigned to the ends of chromosomes A9 and B9, respectively (Figure 1). The pIs of the predicted proteins of AdRAR1 and $A i R A R 1$ were 6.86 and 8.26, respectively, and the MWs were 30.57 and $29.70 \mathrm{kDa}$, respectively (Table 1). Bioinformatics analyses indicated that there are two RAR1 homologs in the allotetraploid cultivated peanut A. hypogaea. Not surprisingly, two $R A R 1$ genes were isolated from the cultivated peanut genome and named $A h R A R 1-A$ and $A h R A R 1-B$. Both deduced amino acid sequences had a molecular weight of $24.5 \mathrm{kDa}$, and their theoretical pIs were 6.81 and 7.11. There were 5 exons in the AhRAR1 sequence and 7 exons in the wild peanut RAR1 sequence (Figure 5A). The RAR1 genes were PCR-amplified from cultivated peanut to analyze their sequences and perform subsequent work. AhRAR1-A and AhRAR1-B shared a similarity of $98.66 \%$, with 3 amino acid differences (Supplementary Figure 1). The encoded proteins were both 224 aa in length, different from the RAR1 proteins in wild peanut. The sequence comparison of RAR1 from cultivated peanut (AhRAR1-A and $A h R A R 1-B)$ and the model plant Arabidopsis (AtRAR1) revealed the same gene structure, as shown in Supplementary Figure 2. Both AhRAR1-A and AtRAR 1 consisted of 6 exons and 5 introns. Notably, AhRAR1 consisted of only 3 base pairs in exon 2 (Supplementary Figures 2A,B). The deduced amino acid of AhRAR1 was composed of three conserved domains: CHORD-I, CHORD-II, and a highly conserved $\mathrm{CCCH}$ domain between the CHORD domains (Supplementary Figure 2C). A phylogenetic tree was constructed based on the RAR1 protein sequence from diverse plant species (Figure 6). The results showed that monocot and dicot RAR1 proteins were separated into two distinct classes (I and II), and the RAR1s from cultivated and wild peanut were distributed among different branches. The phylogenetic analysis results together with the high similarity of amino acid sequences and the same chromosome locations indicated that AdRAR1, AiRAR1, AhRAR1-A1, and AhRAR1-B1 were orthologous genes.

\section{Identification and Characterization of SGT1 Genes}

We predicted 3 SGT1 genes from the A. duranensis and A. ipaensis genomes. They were designated as AdSGT1 to 
TABLE 1 | The HRS-related genes in Arachis genomes.

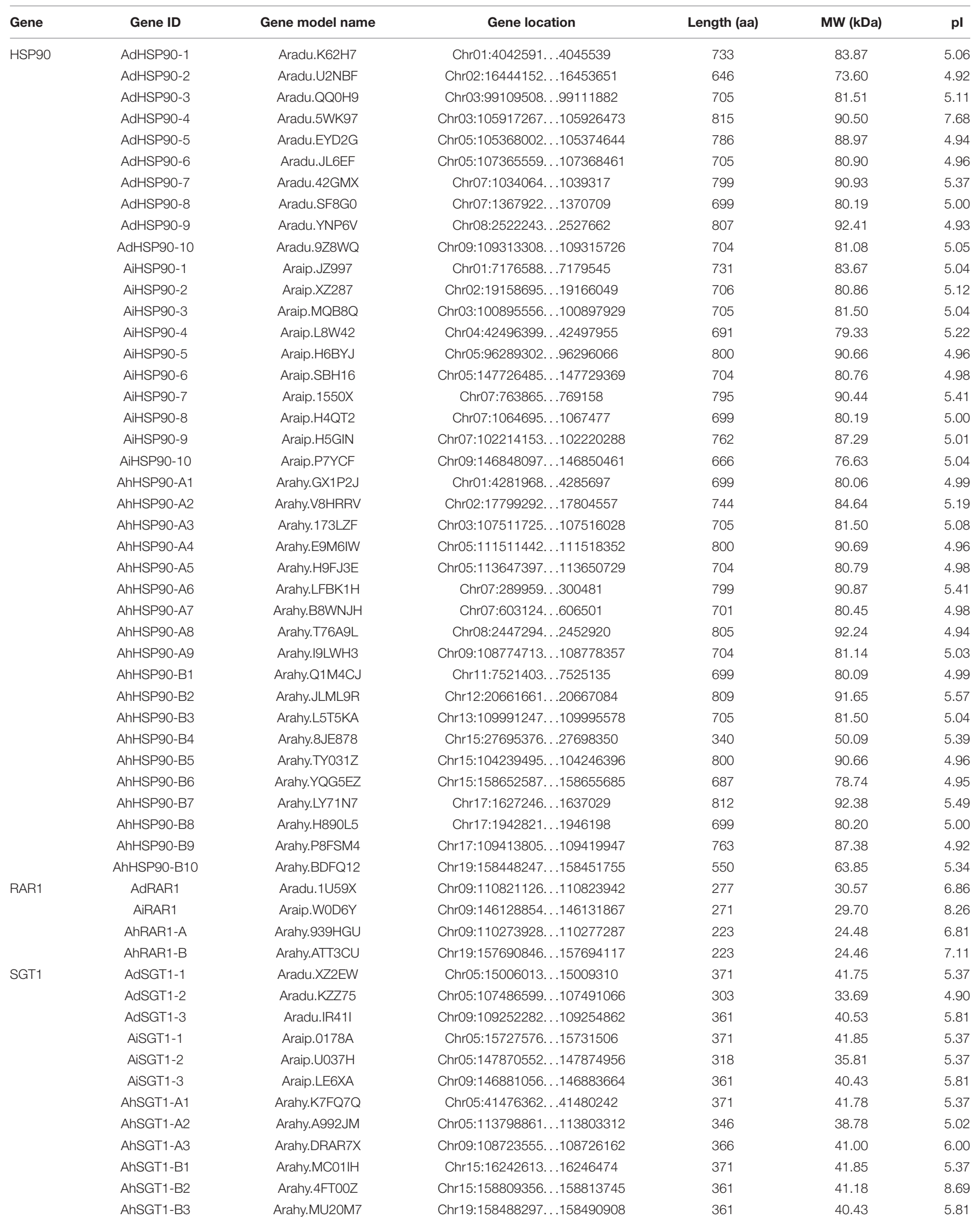



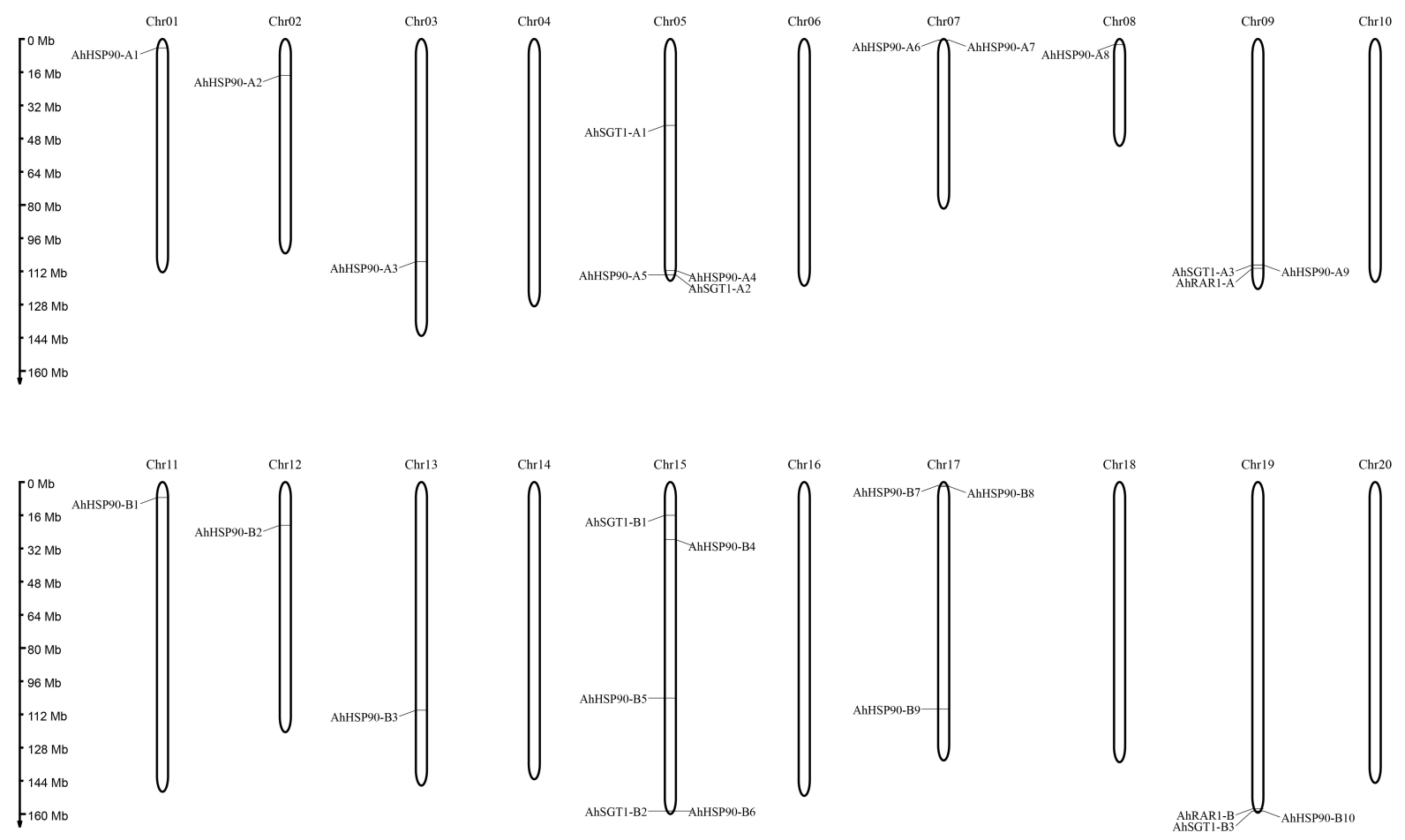

FIGURE 2 | Chromosomal locations of HRS-related genes in the A. hypogaea genome. The chromosome numbers are shown at the top of each chromosome (black bars). The left side of each chromosome corresponds to the approximate location of each HRS-related gene.

TABLE 2 | Orthologous gene pairs in Arachis genomes.

\begin{tabular}{|c|c|c|c|c|c|c|}
\hline A. duranensis & A. ipaensis & A. hypogaea A sub-genome & A. hypogaea B sub-genome & Chromosomal location & CDS identity (\%) & Amino acid identity (\%) \\
\hline AdHSP90-1 & AiHSP90-1 & AhHSP90-A1 & AhHSP90-B1 & A1-B1-1-11 & 99.57 & 99.93 \\
\hline AdHSP90-2 & AiHSP90-2 & AhHSP90-A2 & AhHSP90-B2 & A2-B2-2-12 & 94.98 & 92.63 \\
\hline AdHSP90-5 & AiHSP90-5 & AhHSP90-A4 & AhHSP90-B5 & A5-B5-5-15 & 99.05 & 99.00 \\
\hline AdHSP90-6 & AiHSP90-6 & AhHSP90-A5 & AhHSP90-B6 & A5-B5-5-15 & 98.69 & 99.08 \\
\hline AdHSP90-7 & AiHSP90-7 & AhHSP90-A6 & AhHSP90-B7 & A7-B7-7-17 & 97.48 & 97.61 \\
\hline AdHSP90-10 & AiHSP90-10 & AhHSP90-A9 & AhHSP90-B10 & A9-B9-9-19 & 88.89 & 87.71 \\
\hline AdRAR1 & AiRAR1 & AhRAR1-A & AhRAR1-B & A9-B9-9-19 & 82.65 & 84.93 \\
\hline AdSGT1-1 & AiSGT1-1 & AhSGT1-A1 & AhSGT1-B1 & A5-B5-5-15 & 99.51 & 99.53 \\
\hline AdSGT1-2 & AiSGT1-2 & AhSGT1-A2 & AhSGT1-B2 & A5-B5-5-15 & 90.19 & 85.30 \\
\hline AdSGT1-3 & AiSGT1-3 & AhSGT1-A3 & AhSGT1-B3 & A9-B9-9-19 & 98.14 & 97.88 \\
\hline
\end{tabular}

AdSGT3 and AiSGT1 to AiSGT3 (Table 1). The SGT1 genes identified in $A$. duranensis and A. ipaensis were located on chromosomes 5 and 9, encoded proteins ranging from 303 to 371 amino acid residues in length, and had the highest sequence similarity to the Arabidopsis SGT1 gene (AAL33611.1). The $\mathrm{pI}$ of the predicted proteins ranged from 4.90 to 5.81. Six SGT1 genes were identified from the cultivated peanut genome. We named them AhSGT1-A1 to AhSGT1-A3 and AhSGT1$B 1$ to AhSGT1-B3. They had MWs ranging from 40.24 to $41.85 \mathrm{kDa}$. The encoded proteins varied from 357 to 371 aa in length, with an average of 361 aa (Table 1). Amino acid multiple alignment of the SGT1s from peanut indicated that the AdSGT1-1 and AiSGT1-1 genes were orthologous to AhSGT1A1 and AhSGT1-B1 (98.61\% amino acid identity), and only a few amino acids differed between these genes (Supplementary Figure 3), which were located in the syntenic chromosome location (Figures 1, 2). The AdSGT1-2 and AdSGT1-3 genes were orthologs to AiSGT1-2 and AiSGT1-3, and all were assigned to chromosome 5 (Figure 1). A phylogenetic tree was constructed based on the SGT1 protein sequences from both wild and cultivated peanut. Based on the chromosomal location and phylogenetic analysis results (Figures 1, 5B), this study deduced 


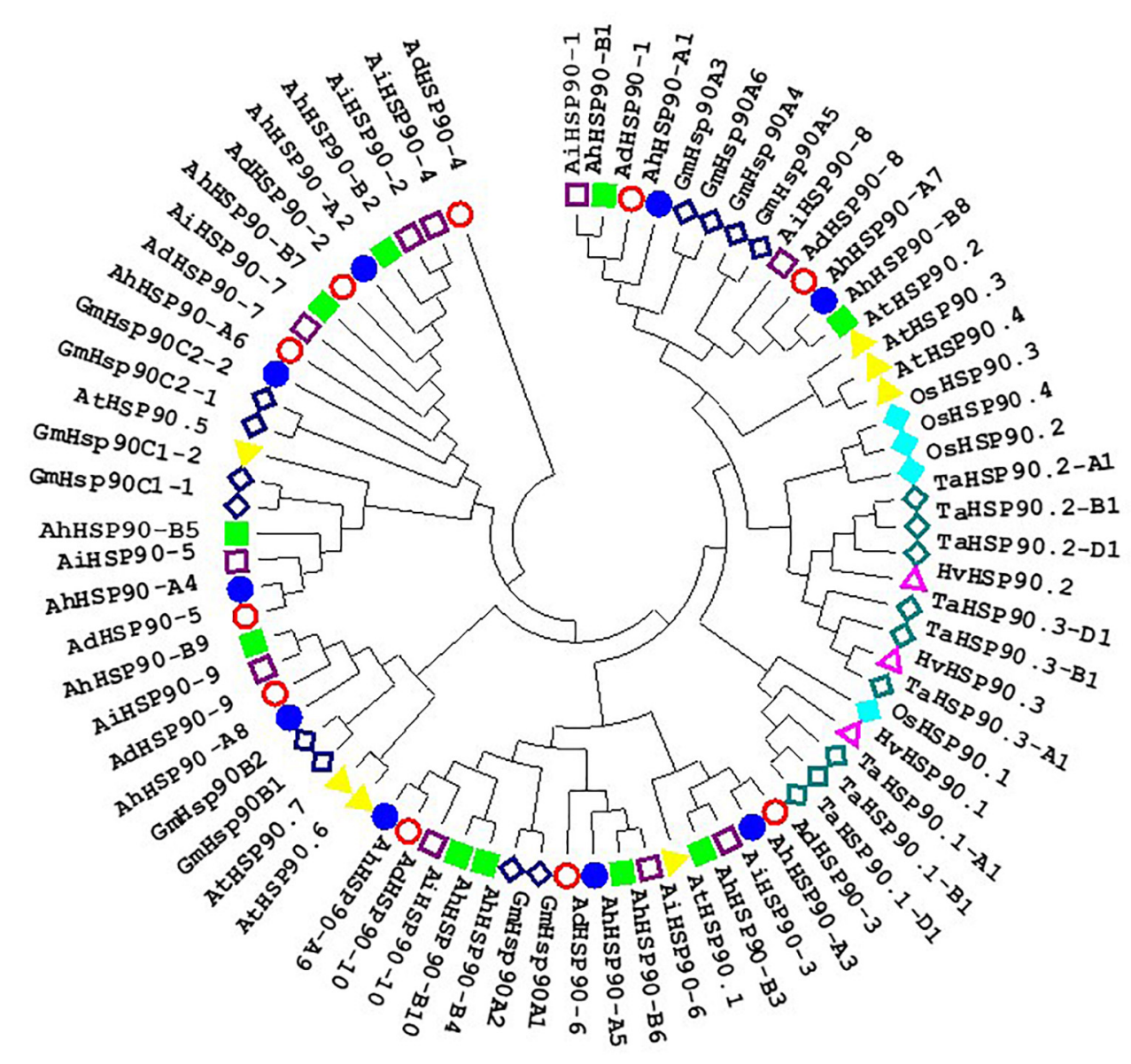

FIGURE 3 | Phylogenetic tree of HSP90 proteins of Arachis, Arabidopsis, rice, soybean, barley, and wheat. Multiple sequence alignment of peanut NAC proteins was performed using ClustalW, and the phylogenetic tree was constructed using MEGA 6.0 by the neighbor-joining method with 1000 bootstrap replicates.

that AhSGT1-A1 and AhSGT1-B1 were orthologous to AdSGT11 and AiSGT1-1, respectively. AhSGT1-A2 and AhSGT1-B2 were orthologous to AdSGT1-2 and AiSGT1-2, and AhSGT1-B2 had the same sequence as AiSGT1-2. AhSGT1-A3 and AhSGT1-B3 were orthologous to AdSGT1-3 and AiSGT1-3 and the previously reported AdSGT1 from $A$. diogoi (Supplementary Figure 4). These orthologous genes shared similar exon-intron structures and conserved motifs (Figure 5B). AdSGT1-1, AdSGT1-3 and their orthologs all consisted of 10 exons, while AdSGT1-2 and its orthologs contained only 9 exons.

\section{Expression Profiling and Responses to Biotic and Abiotic Stresses}

To understand the expression patterns of HRS-related genes, their expression levels in 22 different tissues or developmental stages were examined based on RNA-seq data (Clevenger et al., 2016). We found that orthologous genes exhibited similar expression patterns (for instance, AdHSP90-1 and AiHSP90-1, AdSGT1 and their orthologs in A. ipaensis). Notably, AdRAR1 from $A$. duranensis and AiRAR1 from $A$. ipaensis exhibited the same expression patterns and were constitutively expressed at similar levels in all 22 tissues or developmental stages (Figure 7). AdHSP90-8 and AiHSP90-8 were also expressed in 22 tissues and exhibited higher expression in every tissue than other HRS-related genes, while the expression of AdHSP90-10 and AiHSP90-10 was hardly detected in any tissue. Some genes exhibited tissue-specific expression patterns; for example, AdHSP90-3 and its ortholog AiHSP90-3 exhibited especially high expression in the later seed development stage. Eight genes (AdHSP90-1, AdHSP90-6, AdHSP90-8, and AdHSP909 and their orthologs AiHSP90-1, AiHSP90-6, AiHSP90-8, and AiHSP90-9) were expressed at higher levels than other genes in roots. To identify genes involved in biotic and abiotic stresses, we comparatively reanalyzed RNA-seq data involved in the responses to salt (Zhang et al., 2020), drought (Zhao et al., 2018), low calcium (Chen et al., 2019), and A. flavus infection (Zhao et al., 2020). The results suggested that several HSP90 genes play important roles in salt- and drought-stress responses (Figure 8). AhHSP90-A5, AhHSP90-A9 and $A$ HSP90-B6 were significantly upregulated under salt stress, while $A$ hHSP90-B3 was significantly downregulated. Under drought treatment, AhHSP90-A3, AhHSP90-A5, AhHSP90-A9, AhHSP90-B3, AhHSP90-B6, and AhHSP90-B10 

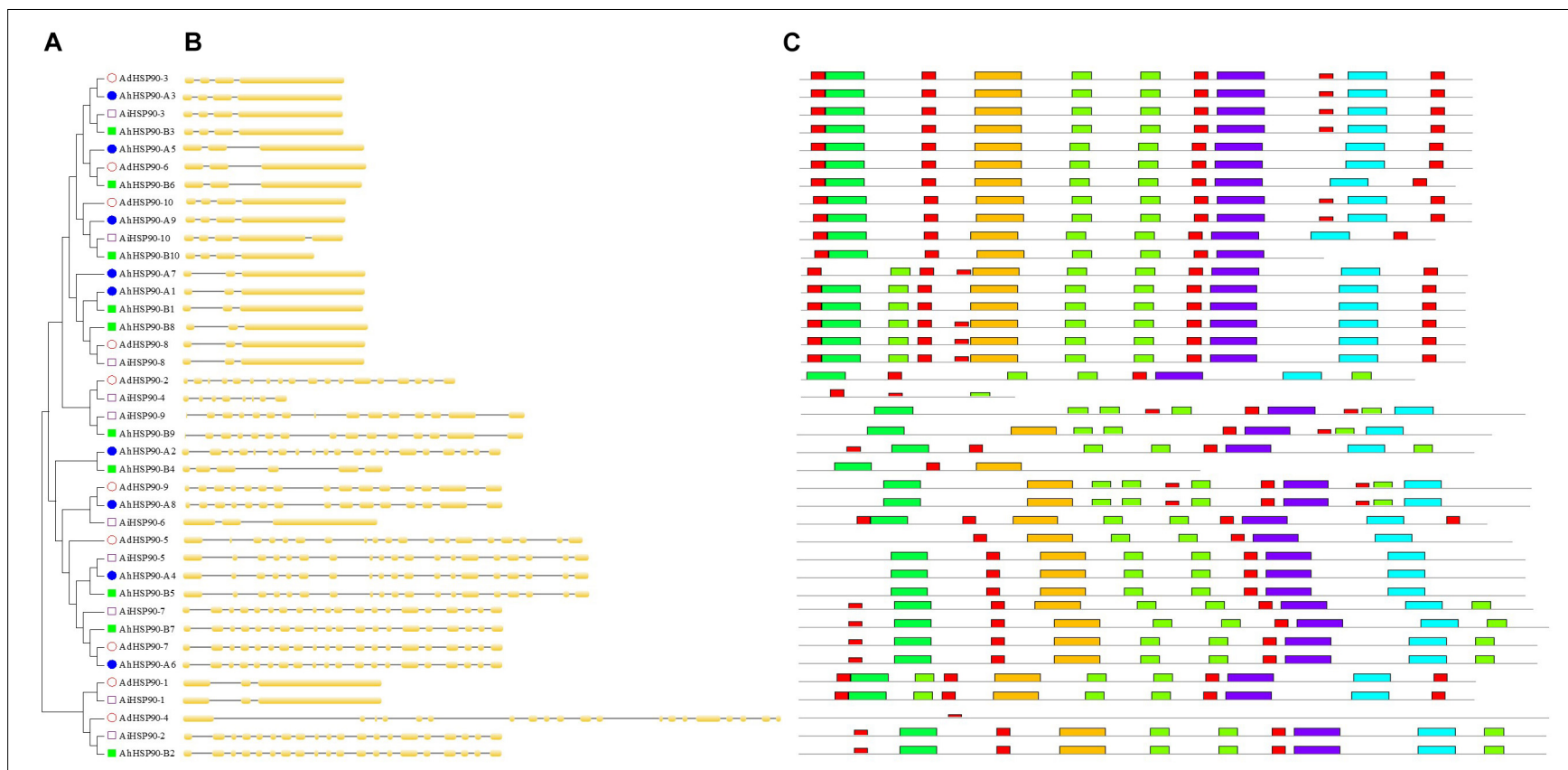

FIGURE 4 | Phylogenetic analysis, gene structures, and conserved motifs of Arachis HSP90 genes. (A) Phylogenetic tree of Arachis HSP90s. (B) Exon-intron structure of Arachis HSP90 genes. (C) Ten conserved motifs in HSP90 proteins.

A

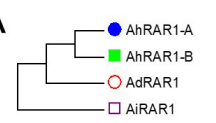

B

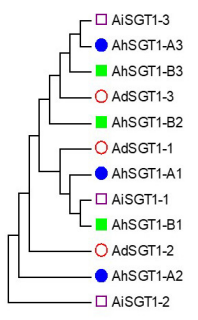

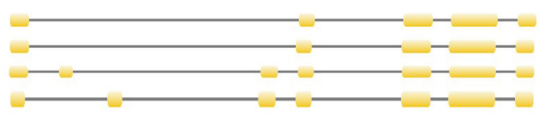
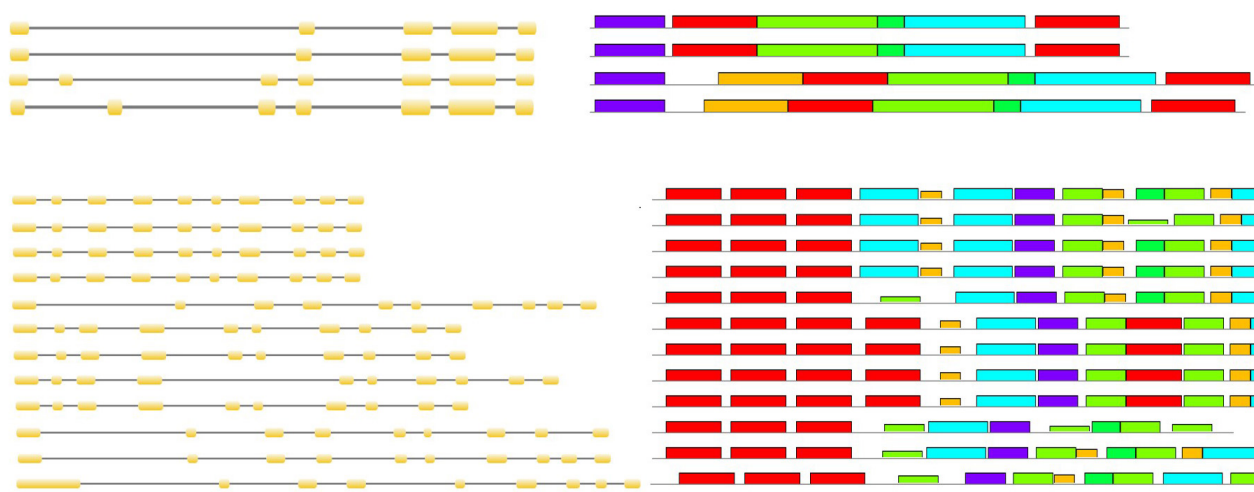

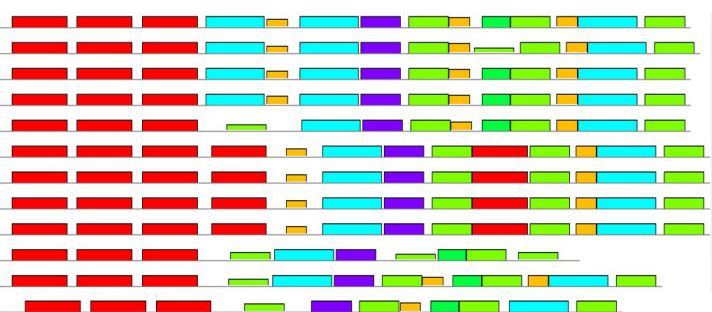

एक ए एम एवा एव

FIGURE 5 | Phylogenetic analysis, gene structures, and conserved motifs of the Arachis RAR1 and SGT1 genes. (A) Arachis RAR1 genes; (B) Arachis SGT1 genes.

were significantly upregulated, and AhHSP90-A6, AhHSP90-A8, and AhHSP90-B9 were significantly downregulated. AhSGT1$B 3$ was upregulated after salt treatment, while AhSGT1-A1 and $A h S G T 1-B 1$ were downregulated. Under drought stress, AhSGT1-A1 and AhSGT1-B1 were both upregulated. Under calcium-deficient conditions, AhSGT1-B3, AhHSP90-A9, and AhHSP90-B10 exhibited higher expression levels than they did under calcium-sufficient conditions, while AhRAR1, AhHSP90-A1, AhHSP90-A7, AhHSP90-A8, AhHSP90-B3, and AhHSP90-B9 exhibited the opposite tendency. The expression levels of AhHSP90-A1, AhHSP90-A7, and AhHSP90-A8 and their orthologous genes AhHSP90-B1, AhHSP90-B7, and AhHSP90-B8 were higher during Aspergillus flavus infection than under the control conditions. AhSGT-A3, AhHSP90-A2, AhHSP90-A3, and AhHSP90-A5 and their orthologous genes
AhSGT-B3, AhHSP90-B2, AhHSP90-B3, and AhHSP90-B6 were downregulated after Aspergillus flavus infection (Figure 8). These orthologous genes exhibited the same expression patterns under stress conditions.

\section{Cloning of RAR1, SGT1, and HSP90 and the Interactions of These Proteins}

To characterize their interactions, AhRAR1-A, AhRAR1-B, AhSGT1-B1, AhSGT1-A2, AhSGT1-B2, AhSGT1-A3, AhSGT1$B 3$, and $A h H S P 90-B 8$ were isolated from the cultivated peanut Huayu 9303 and recombined them into $\mathrm{AD}$ and BD plasmids for $\mathrm{Y} 2 \mathrm{H}$ analysis using the Gal4 System. These 8 genes, when used as prey and bait proteins, showed no or insignificant autoactivation on TDO and QDO media, with the exception 


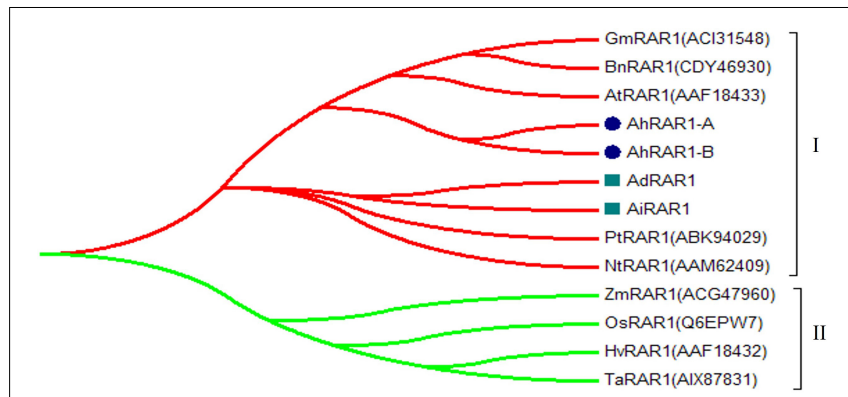

FIGURE 6 | Phylogenetic tree of RAR1 genes from Arachis, Arabidopsis, soybean, barley, common wheat, rice, maize, tobacco, rape, and Populus trichocarpa.

of the AhSGT1-B1 bait on TDO medium (Figure 9). We then tested all possible pairwise interactions among AhRAR1-A, AhRAR1-B, AhSGT1-B1, AhSGT1-A2, AhSGT1-B2, AhSGT1A3, AhSGT1-B3, and AhHSP90-B8 (Figure 10). AhHSP90B8 interacted with AhRAR1s and AhSGT1s in the low stringency medium (TDO) but not in the QDO medium. Strong interaction between AhRAR1s and AhSGT1s were detected, as reflected by yeast growth on the high-stringency medium (QDO) (Figure 10).

\section{DISCUSSION}

HSP90 and its cochaperones RAR1 and SGT1 are usually involved in biotic and abiotic stress responses (Shirasu, 2009; Kadota and Shirasu, 2011). These gene families have been analyzed in several plants, such as Arabidopsis (Krishna and Gloor, 2001), common wheat (Wang et al., 2011, 2015), tobacco (Song et al., 2019), Populus (Zhang et al., 2013), chickpea and pigeonpea (Agarwal et al., 2016). A previous study showed that an SGT1 gene from the wild peanut A. diogoi (AdSGT1) could enhance disease resistance in transgenic tobacco and peanut (Kumar and Kirti, 2015). However, it is still unknown whether HSP90 and RAR1 are expressed in Arachis species. In this study, a systemic analysis of HRS genes in both wild peanut and cultivated peanut were performed.

\section{Identification and Characterization of HRS Genes in Arachis Species}

Ten, 10, and 19 HSP90 genes were retrieved from the genomes of the diploid wild peanut $A$. duranensis, the diploid wild peanut A. ipaensis and the allotetraploid cultivated peanut A. hypogaea, respectively (Table 1). One HSP90 gene was lost in the A subgenome of the allotetraploid cultivated peanut genome, which possibly attributed from incorrect splicing or genome sequencing

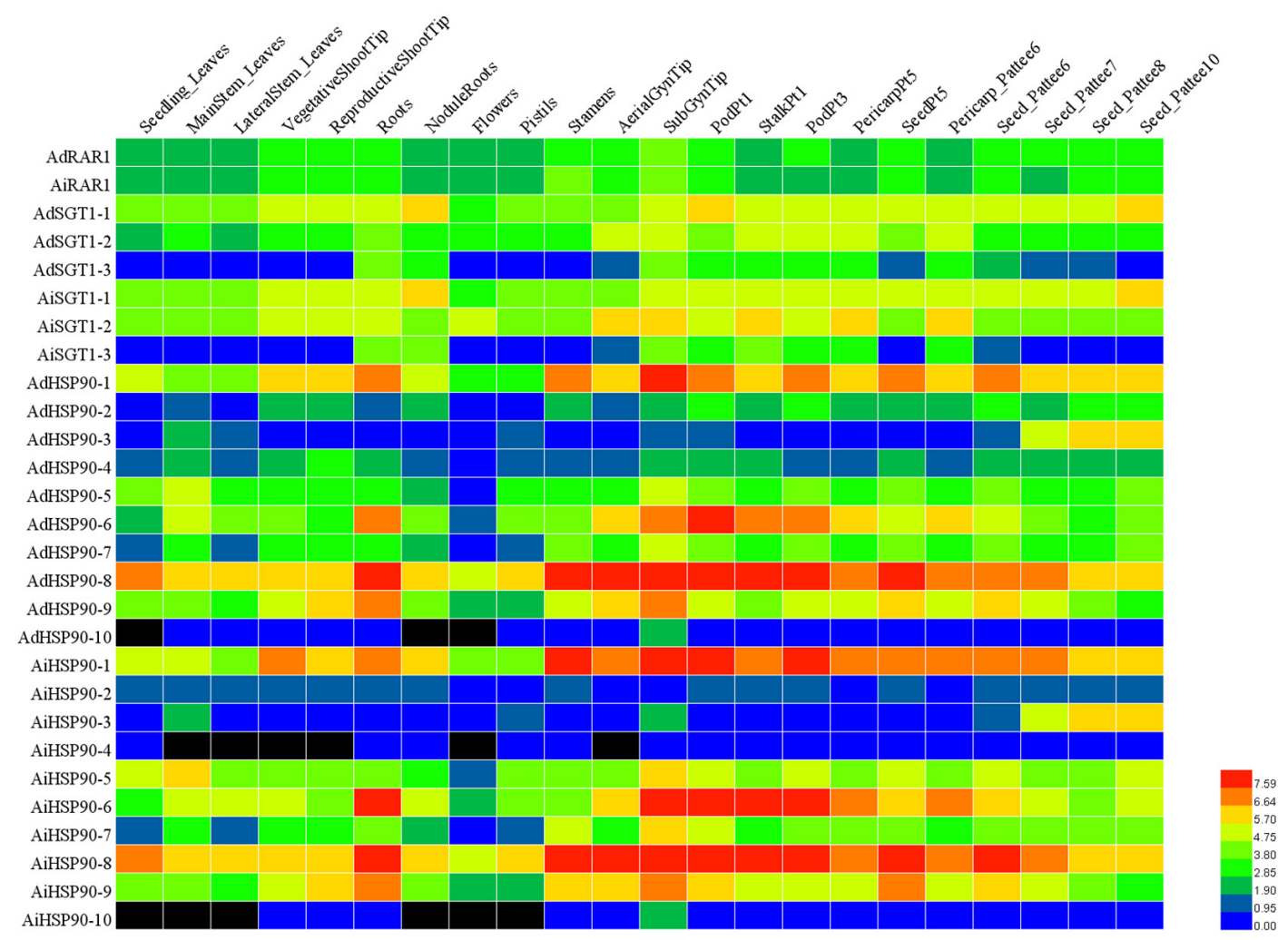

FIGURE 7 | Expression profiling of HRS genes in 22 different tissues of $A$. duranensis and $A$. ipaensis. The FPKM data of each gene were retrieved from Clevenger et al. (2016), log2-transformed and displayed in the form of a heatmap by Heml. The color scale in the lower right indicates relative expression level: green represents a low level, and red indicates a high level. The 22 different tissues are listed at the top of the heat map. The HRS genes are listed on the left of the heatmap. 


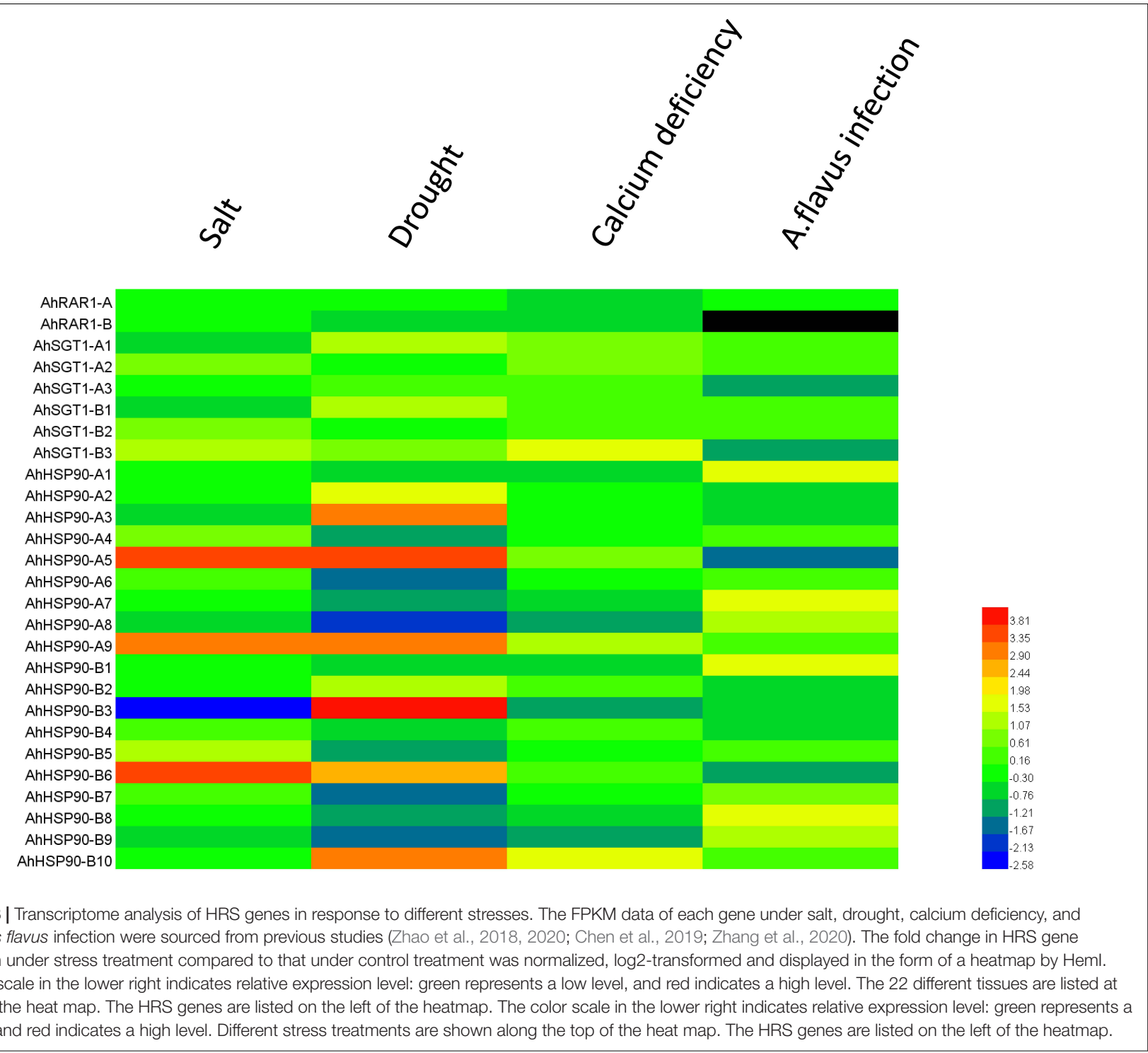

errors. The number of HSP90 genes in cultivated peanut was nearly the sum of that of the two wild peanuts. Similar results were observed for the RAR1 and SGT1 genes, with two RAR1 and six SGT1 genes detected in cultivated peanut and only one RAR1 and three SGT1 genes identified in wild peanut (Table 1). This phenomenon could be attributed to the doubling of chromosomes in diploid wild species during the formation of tetraploid cultivated species (Bertioli et al., 2016; Zhuang et al., 2019). A similar finding was obtained in common wheat, in which the HRS complex has been well studied. There are 3 HSP90 genes in each chromosome of common wheat, that is, nine HSP90 genes in heterohexaploid wheat (AABBDD) (Wang et al., 2011), but only three HSP90 genes in its relative species diploid barley (Pei et al., 2015). There is one RAR1 gene and one SGT1 gene in the barley genome (Pei et al., 2015) and 3 RAR1 and 3 SGT1 genes in the wheat genome (Wang et al., 2011). In plants, the number of HSP90 genes varies greatly. Here, there were nineteen HSP90 genes in the cultivated peanut genome, which is more than the number reported in barely (3) (Pei et al., 2015), wheat (9) (Wang et al., 2011), Arabidopsis (7) (Krishna and Gloor, 2001), rice (9) (Hu et al., 2009), chickpea (5) (Agarwal et al., 2016), pigeonpea (11) (Agarwal et al., 2016), common bean (6) (Agarwal et al., 2016), Medicago (5) (Agarwal et al., 2016), Lotus (5) (Agarwal et al., 2016), and tobacco (11) (Song et al., 2019). This difference may be attributed to cultivated peanut being an allotetraploid plant in which genes have been replicated. These genes share similar intron/exon structures, conserved motifs, and high collinearity chromosomal locations and are considered orthologous genes (Song et al., 2016; Altenhoff et al., 2019). Thirteen orthologous gene pairs in the Arachis genomes were detected in this study (Table 2), for example, AdHSP903, AiHSP90-3, AhHSP90-A3 and AhHSP90-B3 (Figures 3, 4). Phylogenetic analysis showed that the orthologous genes tended to form clusters and shared high sequence similarity (Table 2), which indicated that these orthologous genes might perform the same functions. For example, we speculate that the function of 


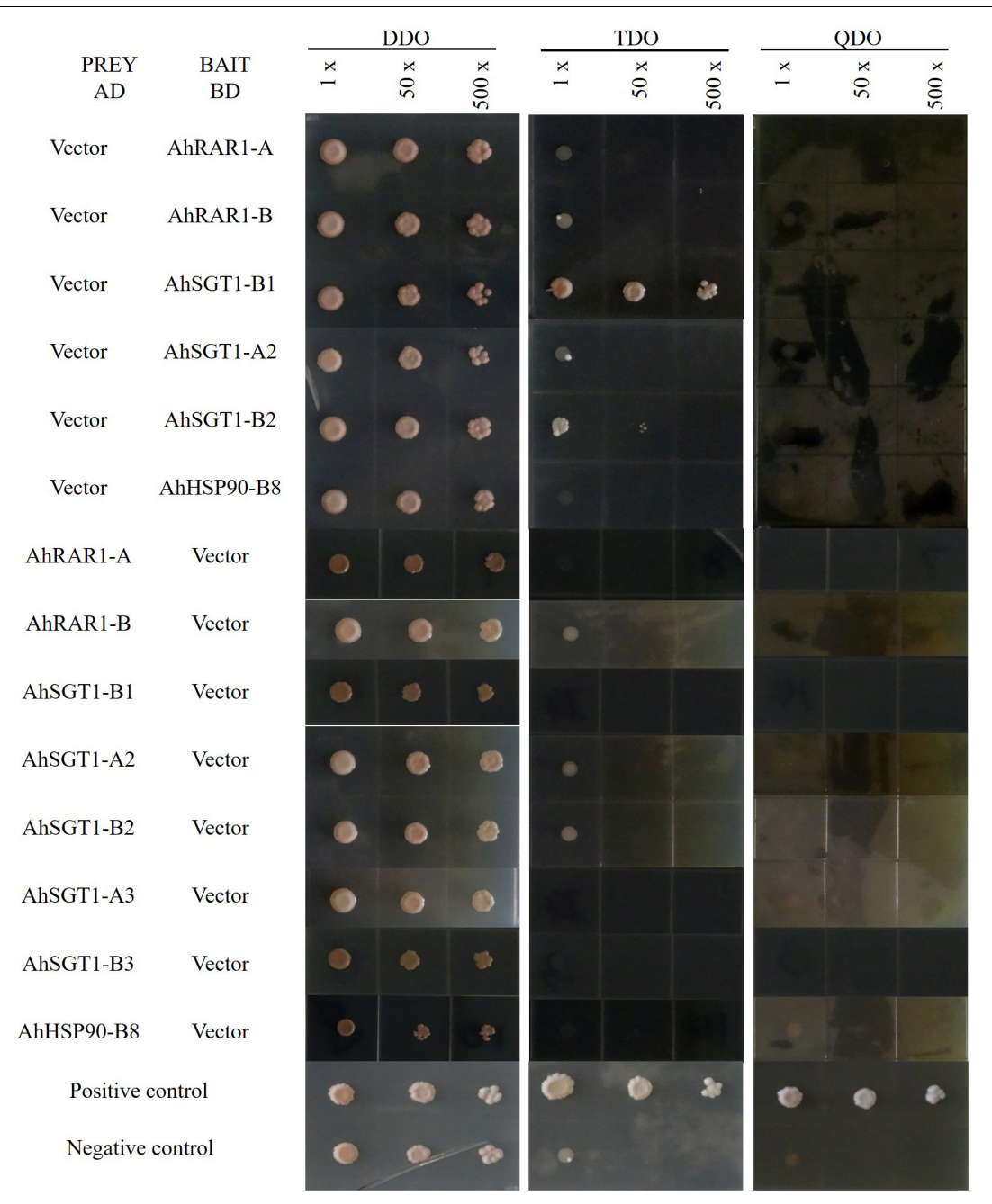

FIGURE 9 | Autoactivation test of HRS proteins. The successfully cotransformed yeast containing both AD and BD plasmids was collected from DDO medium and inoculated on TDO and QDO media with the original resuspension (1x), a 50-fold dilution (50x), or a 500-fold dilution (500x).

AdHSP90-3, AiHSP90-3, AhHSP90-A3, and AhHSP90-B3 is the same as that of AtHSP90.1, which is essential for both abiotic and biotic stress responses in Arabidopsis (Krishna and Gloor, 2001; Haralampidis et al., 2002; Takahashi et al., 2003). Most of the orthologous genes were located on the syntenic locus of each chromosome. The different chromosome locations of orthologous genes AdHSP90-9, AhHSP90-A8, AiHSP90-9, and AhHSP90-B9 suggested that gene locus changes in chromosome locations occurred after divergence and duplication during Arachis evolution. This study found that in the phylogenetic tree, peanut HRS genes tended to cluster with genes from dicotyledons (Figure 3). The HSP90 genes from barley, wheat and rice clustered in the same clade (Figure 3). A similar result was found for RAR1 genes (Figure 6). The phylogenetic results indicated that dicot and monocot HRS genes diverged during evolution, and a similar result was found in tobacco (Song et al., 2019).

The $A h R A R-A$ and $A h R A R 1-B$ fragments were isolated from the cultivated peanut genome. Similar to Arabidopsis
RAR1 (Shirasu et al., 1999), the AhRAR-A and AhRAR1-B sequences each had one 3-bp exon (Supplementary Figure 2), which suggested the same splice patterns in Arabidopsis and peanut. This study found that the sequence of $R A R 1$ from cultivated peanut was quite different from that of wild peanut. RAR1 from wild peanut consisted of two more exons. This result suggested that there were different splice patterns in the wild peanut genome or that incorrect genome assembly occurred. The AhSGT1 genes were isolated from cultivated peanut and found that AhSGT1-A3 and AhSGT1$B 3$ are orthologous genes of the A. diogoi SGT1 gene (Kumar and Kirti, 2015; Supplementary Figure 4), which suggested that AhSGT1-A3 and AhSGT1-B3 represent potential genes for improving leaf late spot disease in peanut. HSP90 usually interacts with its cochaperones RAR1 and SGT1 to perform functions (Wang et al., 2015; Situ et al., 2020). In Arachis, AhRAR-A, AhHSP90-A10, and AhSGT-A3 were clustered together at the end of chromosome 9, and AhRAR-B, AhHSP90$B 10$, and $A h S G T-B 3$ were clustered together at the end 


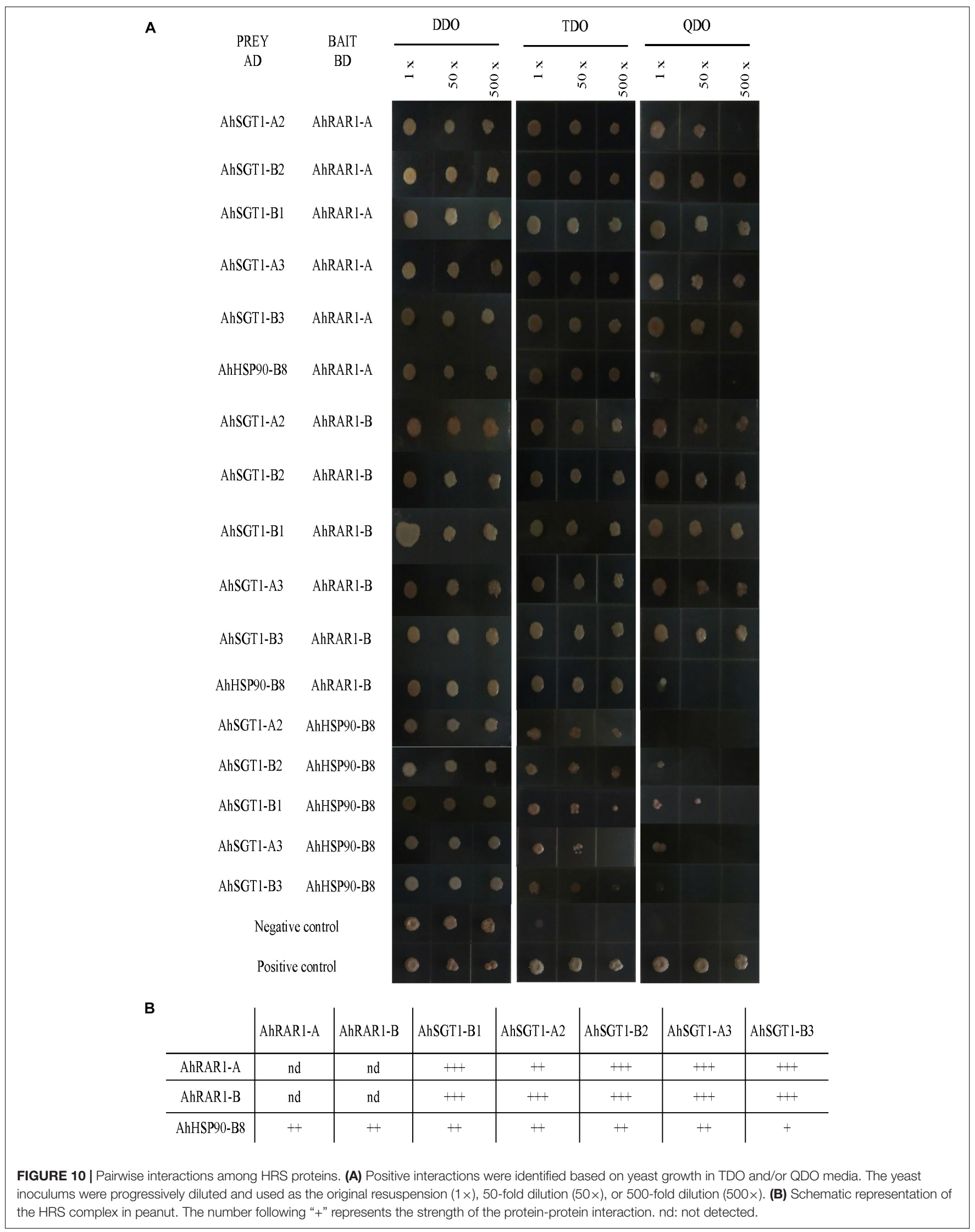


of chromosome 19. The orthologous genes of AhRAR1-A and AhHSP90-A10 might be required for AdSGT1-mediated resistance to leaf late spot disease in the wild peanut $A$. diogoi. In common wheat, all HSP90 genes consist of 3 exons and 2 introns (Wang et al., 2011), while in peanut, the number of exons ranges from 3 to 20 (Figure 4). Only 9 HSP90 genes in the present study contained 3 exons. These results suggest that different evolutionary patterns of HSP90 genes exist between dicots and monocots.

\section{Expression Profiling of HRS Genes in Different Tissues and Stress Conditions}

The expression patterns of genes can provide insights into gene functions (Zhang et al., 2013). RNA-seq is considered a powerful means to study gene expression profiles (Yuan et al., 2019). With the reduction in the cost of RNA-seq, more studies about peanut transcriptome sequencing in different tissues or under different stresses have emerged (Clevenger et al., 2016; Zhao et al., 2018, 2020; Chen et al., 2019; Zhang et al., 2020), which facilitates HRS-related gene expression pattern analysis. The expression patterns of all HRS-related genes were detected based on Clevenger et al. (2016) (Figure 7). This study found that several genes exhibited tissue-specific expression patterns and that their orthologous genes showed similar expression patterns. For example, AdHSP90-10 and its orthologous gene AiHSP90-10 were only expressed in the subterranean gynophore tip (SubGynTip) (Figure 7). The gynophore, which is also called the peg, is a specialized peanut organ that transitions from an upward growth habit to downward outgrowth (subterranean gynophore) upon fertilization (Chen et al., 2016). This study speculate that these two genes might play important roles in the gynophores of subterranean swelling pods. AdHSP901, AdHSP90.6, and AdHSP90.8 were ubiquitously and highly expressed in all 22 tissues that were examined (Figure 7), as were their orthologous genes AiHSP90-1, AiHSP90.6, and AiHSP90.8. These results indicated that these genes play important roles in various physiological activities of peanut. The peanut $R A R 1$ gene was also expressed in all 22 tissues. The expression of AiHSP90-6 in roots was higher than that in leaves and stems, which might indicate its involvement in sodium transport or water absorption in roots to improve peanut salt/drought resistance. As expected, its orthologous gene AhHSP90-B6 was greatly upregulated upon salt or drought treatment (Figure 8). The promoters of those genes that are ubiquitously expressed or that show tissue-specific patterns could be potential tools for peanut quality improvement (Yuan et al., 2019).

Numerous studies have shown that HRS-related genes are involved in the responses to both biotic and abiotic stresses (Liu et al., 2006; Kadota and Shirasu, 2011; Wang et al., 2017). To reveal the responses of HRS-related genes to biotic and abiotic stresses, transcriptome sequencing data of plants under salt, drought, low calcium and A. flavus infection treatments were analyzed (Zhao et al., 2018, 2020; Chen et al., 2019; Zhang et al., 2020). The log2-fold changes under stress treatment are shown in a heatmap (Figure 8). This study found several HRS-related genes involved in the responses to salt, drought, low calcium and A. flavus infection. The expression of AhHSP90-A5, AhHSP90-A9, AhHSP90-B3, and AhHSP90-B6 was significantly upregulated under both salt and drought stresses. However, RAR1 genes seemed to be insignificantly involved in stress responses. This finding implies that Arachis HSP90 genes participate extensively in the responses to salt and drought stress but do not require RAR1. Similarly, previous studies revealed that HSP90 genes were required for R protein-mediated disease resistance in a RAR1- and/or SGT1-independent manner (Hubert et al., 2003; Bhattarai et al., 2007). A previous study showed that Arabidopsis HSP90.7 helped seedlings resist a high concentration of $\mathrm{Ca}^{2+}$ (Chong et al., 2015). In our study, we found that under low calcium conditions, AhHSP90-B10 was upregulated, while AhHSP90-A8 and AhHSP90-B3 were downregulated. The HRS complex is widely involved in R genemediated disease resistance in plants (Shirasu, 2009; Kadota and Shirasu, 2011). Aflatoxin contamination caused by A. flavus is a serious problem for peanut quality (Guo et al., 2009). To reveal the responses of HRS genes to A. flavus, their expression profiles under A. flavus infection were analyzed (Zhao et al., 2020). AhHSP90-A1, AhHSP90-A7, AhHSP90-A8 and their orthologous genes exhibited higher expression levels than other genes under infection, suggesting potential roles of these genes in combatting A. flavus infection. However, the $\mathrm{R}$ gene against $A$. flavus infection stabilized by HSP90 proteins is still unknown. In summary, HRS-related genes, especially HSP90 genes, which are strongly involved in salt and drought stress responses, participate in both biotic and abiotic stress responses.

\section{Interaction Relationship Between HRS Proteins}

Usually, HSP90, RAR1, and SGT1 can interact with each other, and the interactions among them are essential for their functions in plant defense (Shirasu, 2009; Pei et al., 2015). Here, HRS-related genes (including AhRAR1-A, AhRAR1-B, AhSGT1-B1, AhSGT1-A2, AhSGT1-B2, AhSGT1-A3, AhSGT1$B 3$, and $A h H S P 90-B 8$ ) from cultivated peanut were isolated to test their autoactivation and interaction in more detail using a $\mathrm{Y} 2 \mathrm{H}$ assay. When used as prey and bait proteins, all of the detected proteins except the AhSGT1-B1 bait in TDO showed no or insignificant autoactivation on TDO and QDO media (Figure 9). A similar result was observed in barley (Pei et al., 2015), suggesting that AhSGT1-B1 cannot be used as bait for library screening. Previous studies have shown that RAR1 and SGT1 strongly interact with each other in many plant species (Wang et al., 2008, 2015; Fu et al., 2009; Pei et al., 2015). In this study, strong interactions between two AhRAR1 proteins and three AhSGT1 proteins were detected (Figure 10), which might be attributable to the interaction of the CHORD-II domain in RAR1 with the CS domain in SGT1 (Azevedo et al., 2002). AhHSP90-B8 were chose as a representative to investigate its interaction with AhRAR1 and AhSGT1. As in barley (Pei et al., 2015), RAR1 and SGT1 genes in peanut interacted with AhHSP90-B8 in low stringency medium (TDO) (Figure 10). These results indicated that AhRAR1, AhSGT1 and AhHSP90-B8 might perform functions together in peanut. 


\section{DATA AVAILABILITY STATEMENT}

Publicly available datasets were analyzed in this study. This data can be found here: https://www.peanutbase.org/search/gene/.

\section{AUTHOR CONTRIBUTIONS}

CLY, CL, QS, and SS conceived and designed the experiments. CLY, QS, CL, XZ, CXY, and JW performed the experiments and analyzed the data. CLY, QS, and SS wrote the manuscript. All authors read and approved the final manuscript.

\section{FUNDING}

This research was funded by the National Natural Science Foundation of China (32001585 and 31601336), the Shandong Elite Variety Project (2020LZGC001), Taishan Scholar Funding (ts201712080), and the Innovation Project of SAAS (CXGC2021A09).

\section{REFERENCES}

Agarwal, G., Garg, V., Kudapa, H., Doddamani, D., Pazhamala, L. T., Khan, A. W., et al. (2016). Genome-wide dissection of AP2/ERF and HSP90 gene families in five legumes and expression profiles in chickpea and pigeonpea. Plant Biotechnol. J. 14, 1563-1577. doi: 10.1111/pbi.12520

Altenhoff, A. M., Glover, N. M., and Dessimoz, C. (2019). Inferring orthology and paralogy. Methods Mol. Biol. 1910, 149-175. doi: 10.1007/978-1-4939-9074-0_5

Austin, M. J., Muskett, P., Kahn, K., Feys, B. J., Jones, J. D. G., and Parker, J. E. (2002). Regulatory role of SGT1 in early R gene-mediated plant defenses. Science 295, 2077-2080. doi: 10.1126/science.1067747

Azevedo, C., Sadanandom, A., Kitagawa, K., Freialdenhoven, A., Shirasu, K., and Schulze-Lefert, P. (2002). The RAR1 interactor SGT1, an essential component of R gene-triggered disease resistance. Science 295, 2073-2076. doi: 10.1126/ science. 1067554

Bailey, T. L., Mikael, B., Buske, F. A., Martin, F., Grant, C. E., Luca, C., et al. (2009) Meme suite: tools for motif discovery and searching. Nucleic Acids Res. 37, W202-W208.

Bertioli, D. J., Cannon, S. B., Froenicke, L., Huang, G., Farmer, A. D., Cannon, E. K. S., et al. (2016). The genome sequences of Arachis duranensis and Arachis ipaensis, the diploid ancestors of cultivated peanut. Nat. Genet. 48, $438-446$.

Bhattarai, K. K., Li, Q., Liu, Y. L., Dinesh-Kumar, S. P., and Kaloshian, I. (2007). The MI-1-mediated pest resistance requires Hsp90 and Sgt1. Plant Physiol. 144, 312-323. doi: 10.1104/pp.107.097246

Chaudhary, R., Baranwal, K., Kumar, R., Sircar, D., and Chauhan, H. (2019). Genome-wide identification and expression analysis of Hsp70, Hsp90, and Hsp100 heat shock protein genes in barley under stress conditions and reproductive development. Funct. Integr. Genomic 19, 1007-1022. doi: 10.1007/ s10142-019-00695-y

Chen, H., Yang, Q., Chen, K., Zhao, S. S., Zhang, C., and Pan, R. L. (2019). Integrated microRNA and transcriptome profiling reveals a miRNA-mediated regulatory network of embryo abortion under calcium deficiency in peanut (Arachis hypogaea L.). BMC Genomics 20:392. doi: 10.1186/s12864-019-5770-6

Chen, X. P., Yang, Q. L., Li, H. F., Li, H. Y., Hong, Y. B., Pan, L. J., et al. (2016). Transcriptome-wide sequencing provides insights into geocarpy in peanut (Arachis hypogaea L.). Plant Biotechnol. J. 14, 1215-1224. doi: 10.1111/pbi. 12487

Chong, L. P., Wang, Y., Gad, N., Anderson, N., Shah, B., and Zhao, R. M. (2015). A highly charged region in the middle domain of plant endoplasmic reticulum (ER)-localized heat-shock protein 90 is required for resistance to tunicamycin

\section{ACKNOWLEDGMENTS}

The authors appreciate those contributors who made the peanut genome and transcriptome data accessible in public databases.

\section{SUPPLEMENTARY MATERIAL}

The Supplementary Material for this article can be found online at: https://www.frontiersin.org/articles/10.3389/fgene. 2021.689669/full\#supplementary-material

Supplementary Figure 1 | Amino acid comparison between AhRAR1-A and AhRAR1-B.

Supplementary Figure 2 | Gene structure of RAR1 from cultivated peanut. (A) Comparison of DNA sequence between AhRAR1-A, AhRAR1-B and AtRAR1. (B) The numbers and nucleotide acid length of exons and introns of AhRAR1-A, AhRAR1-B and AtRAR1. (C) Deduced amino acid sequence of RAR1. Domains CHORD-I (position 9 to 68), CCCH (100 to 118), and CHORD-II (159 to 218) are boxed. Invariant cysteine and histidine residues are indicated in red letters.

Supplementary Figure 3 | Amino acid comparison among SGT1-1 genes.

Supplementary Figure 4 | Amino acid comparison among SGT1-3 genes.

or high calcium-induced ER stresses. J. Exp. Bot. 66, 113-124. doi: 10.1093/jxb/ eru403

Clevenger, J., Chu, Y., Scheffler, B., and Ozias-Akins, P. (2016). A developmental transcriptome map for allotetraploid Arachis hypogaea. Front. Plant Sci. 7:1446. doi: $10.3389 /$ fpls. 2016.01446

Dash, S., Cannon, E. K. S., Kalberer, S. R., Farmer, A. D., and Cannon, S. B. (2016). "Chapter 8 - peanutBase and other bioinformatic resources for peanut," in Peanuts, eds H. T. Stalker and R. F. Wilson (Urbana, IL: AOCS Press), 241-252,

Deng, W. K., Wang, Y. B., Liu, Z. X., Cheng, H., and Xue, Y. (2014). HemI: a toolkit for illustrating heatmaps. PLoS One 9:e111988. doi: 10.1371/journal. pone. 0111988

Fu, D. Q., Ghabrial, S., and Kachroo, A. (2009). GmRAR1 and GmSGT1 are required for basal, $\mathrm{R}$ gene-mediated and systemic acquired resistance in soybean. Mol. Plant Microbe Interact. 22, 86-95. doi: 10.1094/mpmi-22-1-0086

Guo, B. Z., Yu, J. J., Holbrook, C. C., Cleveland, T. E., Nierman, W. C., and Scully, B. T. (2009). Strategies in prevention of preharvest aflatoxin contamination in peanuts: aflatoxin biosynthesis, genetics and genomics. Peanut Sci. 36, 11-20. doi: 10.3146/at07-001.1

Haralampidis, K., Milioni, D., Rigas, S., and Hatzopoulos, P. (2002). Combinatorial interaction of cis elements specifies the Expression of the Arabidopsis AtHsp90-1 Gene. Plant Physiol. 129, 1138-1149. doi: 10.1104/pp.004044

Hu, B., Jin, J., Guo, A. Y., Zhang, H., Luo, J., and Gao, G. (2015). GSDS 2.0: an upgraded gene feature visualization server. Bioinformatics 31, 1296-1297. doi: 10.1093/bioinformatics/btu817

Hu, W. H., Hu, G. C., and Han, B. (2009). Genome-wide survey and expression profiling of heat shock proteins and heat shock factors revealed overlapped and stress specific response under abiotic stresses in rice. Plant Sci. 176, 583-590. doi: 10.1016/j.plantsci.2009.01.016

Hubert, D. A., Tornero, P., Belkhadir, Y., Krishna, P., Takahashi, A., Shirasu, K., et al. (2003). Cytosolic HSP90 associates with and modulates the Arabidopsis RPM1 disease resistance protein. EMBO J. 22, 5679-5689. doi: 10.1093/emboj/ cdg547

Ito, M., Ohnishi, K., Hikichi, Y., and Kiba, A. (2015). Molecular chaperons and cochaperons, Hsp90, RAR1, and SGT1 negatively regulate bacterial wilt disease caused by Ralstonia solanacearum in Nicotiana benthamiana. Plant Signal Behav. 10:970410.

Kadota, Y., and Shirasu, K. (2011). The HSP90 complex of plants. Biochim. Biophys. Acta 1823, 689-697. doi: 10.1016/j.bbamcr.2011.09.016

Krishna, P., and Gloor, G. (2001). The Hsp90 family of proteins in Arabidopsis thaliana. Cell Stress Chaperones 6, 238-246. doi: 10.1379/1466-1268(2001) 006<0238:thfopi $>2.0$. co; 2 
Kumar, D., and Kirti, P. B. (2015). Pathogen-induced SGT1 of Arachis diogoi induces cell death and enhanced disease resistance in tobacco and peanut. Plant Biotechnol. J. 13, 73-84. doi: 10.1111/pbi.12237

Larkin, M. A., Blackshields, G., Brown, N. P., Chenna, R., McGettigan, P. A., McWilliam, H., et al. (2007). Clustal W and clustal X version 2.0. Bioinformatics 23, 2947-2948. doi: 10.1093/bioinformatics/btm404

Liu, D., Zhang, X., Cheng, Y., Takano, T., and Liu, S. (2006). rHsp90 gene expression in response to several environmental stresses in rice (Oryza sativa L.). Plant Physiol. Bioch. 44, 380-386. doi: 10.1016/j.plaphy.2006.06.011

Pei, H. C., Sun, Q. X., Hao, Q. Q., Lv, B., Wu, J. J., and Fu, D. L. (2015). The HSP90RAR1-SGT1 based protein interactome in barley and stripe rust. Physiol. Mol. Plant P. 91, 11-19. doi: 10.1016/j.pmpp.2015.02.004

Reddy, P. S., Thirulogachandar, V., Vaishnavi, C. S., Aakrati, A., Sopory, S. K., and Reddy, M. K. (2011). Molecular characterization and expression of a gene encoding cytosolic Hsp90 from Pennisetum glaucum and its role in abiotic stress adaptation. Gene 474, 29-38. doi: 10.1016/j.gene.2010.12.004

Shanmugam, A., Thamilarasan, S. K., Park, J. I., Jung, M. Y., and Nou, I. S. (2016). Characterization and abiotic stress-responsive expression analysis of SGT1 genes in Brassica oleracea. Genome 59, 243-251. doi: 10.1139/gen-2015-0128

Shirasu, K. (2009). The HSP90-SGT1 chaperone complex for NLR immune sensors. Annu. Rev. Plant Biol. 60, 139-164. doi: 10.1146/annurev.arplant.59.032607. 092906

Shirasu, K., Lahaye, T., Tan, M. W., Zhou, F., Azevedo, C., and Schulze-Lefert, P. (1999). A novel class of eukaryotic zinc-binding proteins is required for disease resistance signaling in barley and development in C. Elegans. Cell 99, 355-366. doi: 10.1016/s0092-8674(00)81522-6

Situ, J. J., Jiang, L. Q., Fan, X. N., Yang, W. S., Li, W., Xi, P. P., et al. (2020). An RXLR effector PlAvh142 from Peronophythora litchii triggers plant cell death and contributes to virulence. Mol. Plant Pathol. 21, 415-428. doi: 10.1111/mpp. 12905

Song, H., Wang, P. F., Lin, J. Y., Zhao, C. Z., Bi, Y. P., and Wang, X. J. (2016). Genome-wide identification and characterization of WRKY gene family in peanut. Front. Plant Sci. 7:534. doi: 10.3389/fpls.2016.00534

Song, Z. P., Pan, F. L., Yang, C., Jia, H. F., Jiang, H. L., He, F., et al. (2019). Genomewide identification and expression analysis of HSP90 gene family in Nicotiana tabacum. BMC Genet. 20:35. doi: 10.1186/s12863-019-0738-8

Takahashi, A., Casais, C., Ichimura, K., and Shirasu, K. (2003). HSP90 interacts with RAR1 and SGT1 and is essential for RPS2-mediated disease resistance in Arabidopsis. Proc. Natl. Acad. Sci. U.S.A. 100, 11777-11782. doi: 10.1073/pnas. 2033934100

Tamura, K., Stecher, G., Peterson, D., Filipski, A., and Kumar, S. (2013). MEGA6:molecular evolutionary genetics analysis version 6.0. Mol. Bio. Evol. 30, 2725-2729. doi: 10.1093/molbev/mst197

Vishwakarma, H., Junaid, A., Manjhi, J., Singh, G. P., Gaikwad, K., and Padaria, J. C. (2018). Heat stress transcripts, differential expression, and profiling of heat stress tolerant gene TaHsp90 in Indian wheat (Triticum aestivum L.) cv C306. PLoS One 13:e0198293. doi: 10.1371/journal.pone.0198293

Wang, G. F., Fan, R. C., Wang, X. P., Wang, D. W., and Zhang, X. Q. (2015). TaRAR1 and TaSGT1 associate with TaHsp90 to function in bread wheat (Triticum aestivum L.) seedling growth and stripe rust resistance. Plant Mol. Biol. 87, 577-589. doi: 10.1007/s11103-015-0298-x

Wang, G. F., Wei, X. N., Fan, R. C., Zhou, H. B., Wang, X. P., Yu, C. M., et al. (2011). Molecular analysis of common wheat genes encoding three types of cytosolic heat shock protein 90 (Hsp90): functional involvement of cytosolic Hsp90s in the control of wheat seedling growth and disease resistance. New Phytol. 191, 418-431. doi: 10.1111/j.1469-8137.2011.03715.x

Wang, X. J., Wang, Y. R., Liu, P., Ding, Y., Mu, X. Q., Liu, X. P., et al. (2017). TaRar1 is involved in wheat defense against stripe rust pathogen mediated by YrSu. Front. Plant Sci. 8:156. doi: 10.3389/fpls.2017.00156

Wang, Y. L., Gao, M. J., Li, Q., Wang, L. Y., Wang, J. J., Jeon, J. S., et al. (2008). OsRAR1 and OsSGT1 physically interact and function in rice basal disease resistance. Mol. Plant Microbe Interact. 21, 294-303. doi: 10.1094/mpmi-213-0294

Yuan, C. L., Sun, Q. X., and Kong, Y. Z. (2019). Genome-wide mining seed-specific candidate genes from peanut for promoter cloning. PLoS One 14:e0214025. doi: 10.1371/journal.pone.0214025

Zhang, H., Zhao, X. B., Sun, Q. X., Yan, C. X., Wang, J., Yuan, C. L., et al. (2020). Comparative transcriptome analysis reveals molecular defensive mechanism of Arachis hypogaea in response to salt stress. Int. J. Genomics 2020:6524093.

Zhang, J., Li, J. B., Liu, B. B., Zhang, L., Chen, J., and Lu, M. Z. (2013). Genomewide analysis of the Populus Hsp90 gene family reveals differential expression patterns, localization, and heat stress responses. BMC Genomics 14:532. doi: 10.1186/1471-2164-14-532

Zhang, M., Shen, Z. W., Meng, G. Q., Lu, Y., and Wang, Y. L. (2017). Genomewide analysis of the Brachypodium distachyon (L.) P. Beauv. Hsp90 gene family reveals molecular evolution and expression profiling under drought and salt stresses. PLoS One 12:e0189187. doi: 10.1371/journal.pone.0189187

Zhao, C. Z., Li, T. T., Zhao, Y. H., Zhang, B. H., Li, A. Q., Zhao, S. Z., et al. (2020). Integrated small RNA and mRNA expression profiles reveal miRNAs and their target genes in response to Aspergillus flavus growth in peanut seeds. BMC Plant Biol. 20:215. doi: 10.1186/s12870-020-02426-z

Zhao, X. B., Li, C. J., Wan, S. B., Zhang, T. T., and Shan, S. S. (2018). Transcriptomic analysis and discovery of genes in the response of Arachis hypogaea to drought stress. Mol. Bio. Rep. 45, 119-131. doi: 10.1007/s11033-018-4145-4

Zhuang, W. J., Chen, H., Yang, M., Ramchiary, N., Pandey, M. K., Zhang, C., et al. (2019). The genome of cultivated peanut provides insight into legume karyotypes, polyploid evolution and crop domestication. Nat. Genet. 51, 865876.

Conflict of Interest: The authors declare that the research was conducted in the absence of any commercial or financial relationships that could be construed as a potential conflict of interest.

Publisher's Note: All claims expressed in this article are solely those of the authors and do not necessarily represent those of their affiliated organizations, or those of the publisher, the editors and the reviewers. Any product that may be evaluated in this article, or claim that may be made by its manufacturer, is not guaranteed or endorsed by the publisher.

Copyright (c) 2021 Yuan, Li, Zhao, Yan, Wang, Mou, Sun and Shan. This is an open-access article distributed under the terms of the Creative Commons Attribution License (CC BY). The use, distribution or reproduction in other forums is permitted, provided the original author(s) and the copyright owner(s) are credited and that the original publication in this journal is cited, in accordance with accepted academic practice. No use, distribution or reproduction is permitted which does not comply with these terms. 